\title{
Current State of Nanofiber Produced by Electrospinning and Prospects for Mass Production
}

\author{
YAMASHITA Yoshihiro \\ Materials Science, The University of Shiga Prefecture, \\ 2500 Hassaka, Hikone, Shiga 522-8533, Japan
}

Received 20 October 2008; accepted for publication 7 November 2008

\begin{abstract}
Melt electrospinning was able to achieve a thin fiber with nano-order diameter by controlling the amount of discharge. Biomaterial is one of the fields where nanofiber is actively studied. The challenge of Japan for application and mass production of nanofiber noticed at present is also explained in this paper. Hirose paper Co. Ltd. proposed the production technique for an electrospinning in bubbles which continuously arise in polymer solution by applying high voltage. In NEDO project of Japan, the productivity was improved by increasing number of nozzle and became 50 times as great as the previous one. In Shiga Prefecture, the manufacturing equipment was also developed.
\end{abstract}

Key Words: Electrospinning, Nanofiber, Multi nozzle, Melt electrospinning

\section{Introduction}

Nanofiber produced through an electrospinning process is widely utilized in medical filters, electronic materials, etc. [1]. Moreover, manufacture of electrospinning machines of a solution-spun type has started in Japan as well. This paper describes the challenge in Japan for the application and mass production of nanofiber, which have been receiving so much attention recently.

\section{Melt electrospinning}

Electrospinning equipment of the solvent-free melting method has been receiving a considerable amount of attention, and research on electrospinning using lasers is detailed in the report of Ogata et al. [2-4] of Fukui University and Ohkoshi et al. [5-7] of Shinshu University. Here, the research of Dalton et al. [8] is introduced. Diameter of the fiber produced through the melt electrospinning only from polypropylene resin (PP-15, Bayer AG, MFI $\left.15 \mathrm{~cm}^{3} / 10 \mathrm{~min}\left(230^{\circ} \mathrm{C} / 2.16 \mathrm{~kg}\right)\right)$ were $35.6 \mu \mathrm{m}$. However, through the addition of the polypropylene modifier IRGATEC CR76 (Ciba-Geigy) to $1.5 \%$ in the solution, the fiber diameter decreased to $840 \mathrm{~nm}$. This modifier is radical generator agent which controls a decrease in molecular weight of the polypropylene resin.
This is used to produce melt-blow, non-woven fabric using spun-bound grade resin, and improves the property of the barrier and the mechanical characteristic for the obtained nonwoven fibers. This additive has the possibility of thinning the fiber diameter by lowering the viscosity of the resin, however, it has also the possibility of polymer chain breakage.

Moreover, the melt electrospinning of the blend of polycaprolactone PCL (The molecular weight was $67000 \mathrm{~g} / \mathrm{mol}$ ) and copolymer PEG47-b-PCL95 of polyethylene glycol PEG and PCL were examined. Melt electrospinning becomes possible by blending the copolymer in PCL. Melt electrospun nanofiber was able to produce a thin fiber diameter by curbing the amount of discharge to $0.05 \mathrm{ml} / \mathrm{h}$. The authors tried a further experiment using the generalpurpose melt spinning equipment. In the melt electrospinning of low molecular weight PP, a fiber diameter of 1-5 $\mu \mathrm{m}$ was obtained (Fig. 1).

Research into the melt blow was carried out by Ellison et al. [9]. A fiber averaging $300 \mathrm{~nm}$ was obtained by adjusting the melt temperature, blow temperature, and gas velocity along with the quantity of delivery using melt-blow grade PP (isotactic PP3746G, Exxon; MFR 1500 (230 $\left.{ }^{\circ} \mathrm{C}\right)$, Mn: 10600, Mw: 59000). The distribution of the fiber diameter varied from 100 to $2000 \mathrm{~nm}$. Because melting time was as long as $30 \mathrm{~min}$, however, Mn and Mw decreased to $38 \%$ and

Corresponding author: E-mail: yamashit@mat.usp.ac.jp, Tel/Fax: +81-749-28-8582 


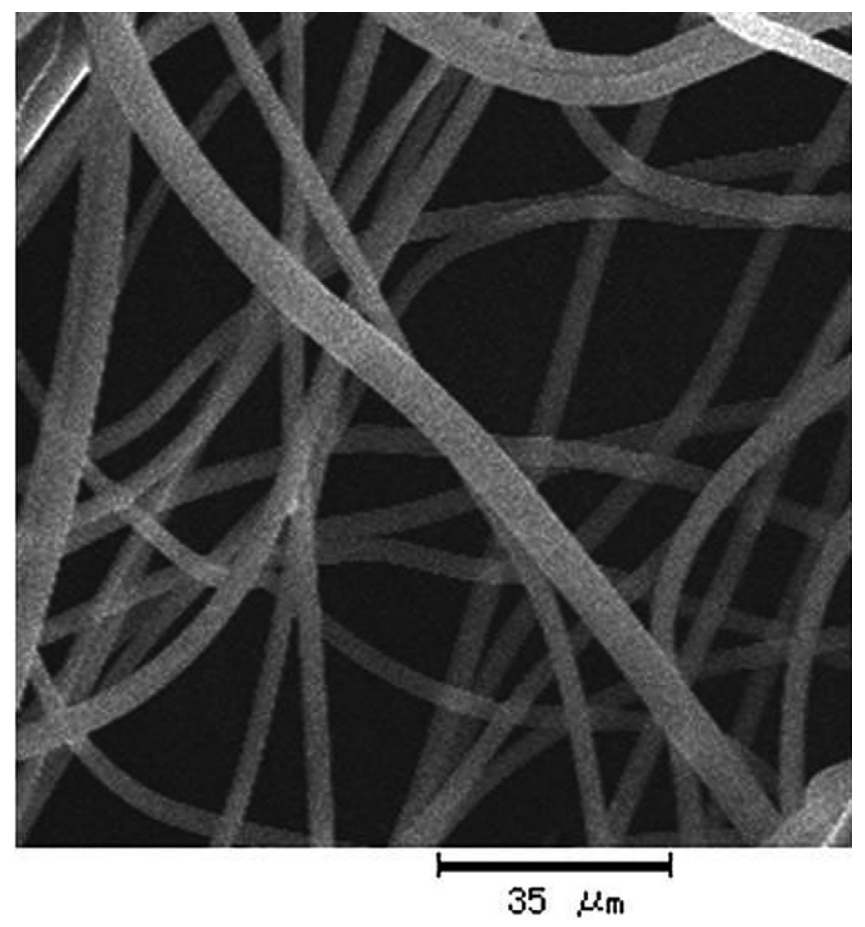

Fig. 1 Melt electrospinning fiber of PP.

$51 \%$, respectively. Ellison asserted that in order to attain nanofiber with the melt-blow technique, it is crucial to have elasticity such that the fiber would not be severed through the friction by way of the blow process rather than the demanded the entanglement of the polymer chain.

\section{Electronic material}

Chi-Ching Kuo et al. [10] produced nanofiber with PMMA blended with various polyfluorenes (Fig. 2). The fibers obtained from the blending of PFTP/PMMA along with

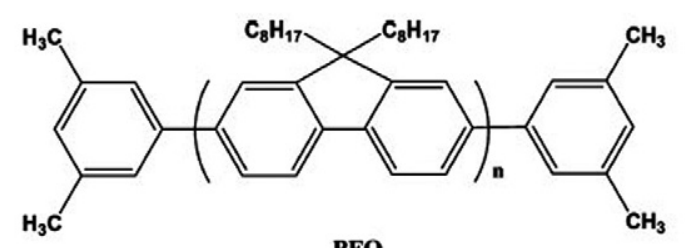

PFO

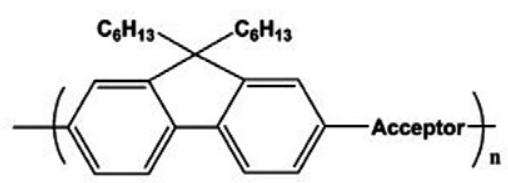

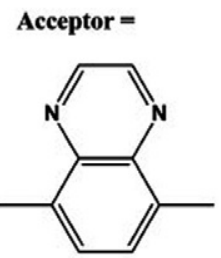

PFQ

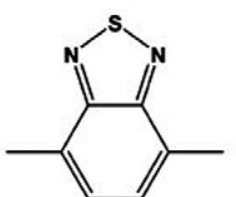

PFBT

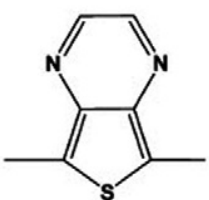

PFTP
Fig. 2 Organic luminescence material [10].
PFO/PMMA, PFQ/PMMA, and PFBT/PMMA respectively showed the respective color luminescence of red, yellow, green, and blue. The luminescence efficiency exceeded that of spincasted film. The polyfluorenes were added to $1 \sim 50 \mathrm{wt} \%$ in PMMA (with a molecular weight of 350000). The diameter of each nanofiber ranged from 250 to $750 \mathrm{~nm}$. The dispersal of polyfluorene into the fiber occurs when the amount of additives is low, however, when the amount of polyfluorene is increased a core shell structure is formed to create a sequential aggregate structure in the center of the fiber. This aggregate structure showed a higher luminescence efficiency than that of spin-coated products.

\section{Biomaterial}

Biomaterial is currently one of the most researched and utilized fields of nanofiber production. The nanofiber obtained by spinning from a 50-50 blend of chloroform fluid of PHB (poly (3-hydroxybutyrate), PHBV (poly (3hydroxybutyrate-co-2-hydroxyvalerate)) excelled in adhesion and the cultivation of a cells in comparison to that obtained from cast film [11]. The nanofiber mat from the 50-50 blend of w/w PHB/PHBV mixed solutions presented the highest ALP potency in these. These results signify the high potential for the use of these electrospinning nanofiber mats as bone-tissue scaffolds.

Santi Tungprapa et al. [12] compared various model medicines (naproxen (NAP), indometacin (IND), ibuprofen (IBU), sulindac (SUL) ) contained in calcium acetate (CA) nano fiber and their drug release characteristics with those of cast film. For each drug, a $20 \mathrm{wt} \%$ was added against the CA. The obtained nanofiber diameter was 263-297 nm. This nanofiber exhibited a quicker emission characteristic than cast film with all medicines.

Sakai et al. [13] proposed the scaffold of nanofiber through the electrospinning process. With the calcination of silica nano-nanofiber (50-800 $\mathrm{nm}$ ) obtained by the electrospinning of tetraethoxysilane at $500^{\circ} \mathrm{C}$ as a starting material, they discovered that cells adhere to the fiber extremely effectively. It can be surmised that this is due to the improved adhesive properties between carrier and cell, because the various secreted proteins by the cells adhere more easily to the hydrophobic surface. Further, an MG63 osteoblast was cultured on silica nanofiber (uncalcinated). As a result, cells were cultivated along the distributed length of the fiber.

\section{Manufacturing of nanofiber}

\section{1 Present state of the world}

Donaldson Co. Inc. [14] produces automobile oil filters by entrusting their manufacture to companies like E-spin [15] 
etc.. E-spin engages in the continuous production of nonwoven nanofiber of a width of 64 inches. These are quite likely produced through the nozzle system. Finetex Technology Global (Hong Kong, Korea) [16] carries out the sale and processing on consignment of a products coated with nanofibers produced with electrospinning on nonwoven fabrics and products produced by sandwiching nanofiber between nonwoven fabric. Their factories are in Philippines. They have also decreased the nozzle system. Awa Paper MFG. Co. Ltd. (Japan) has been offering articles jointly developed with Finetex Technology in the market since August, 2008. As a filter medium for vehicle engines and gas turbines, it offers a product with a nanofiber coating on the filter paper surface. The nanofiber processing technology of a Finetex Technology was combined with technological know-how of the filter medium for engines from Awa paper MFG [17]. With air filters, it is said that there is about a $50 \%$ improvement in ultra-fine, particle-capture performance such as the carbon dust and the double of durability were achieved in comparison with conventional filter mediums for the engines.

Nanotechnics (South Korea) [18] is a company in South Korea which Dupont purchased in 2002. Though they are developing nanofiber which excels in heat-resistance, it is not clear presently which specific products are employed. Nanostatics (USA) [19] was a venture-capital, nanofiberprocessing company in Ohio, and it offered the coating of nanofiber through electrospinning through their proprietary technology. They are able to produce nanfibers of a width of $60 \mathrm{~cm}$ at a speed of $6 \mathrm{~m}$ per minute or more through fluid and electrostatic field control.

Furthermore, I.S. Kim of Shinshu University has developed a large-scale electrospinning device for trial purposes. It has produced nanofiber samples of a $60 \mathrm{~cm}$ width and $2 \mathrm{~m}$ length using a total of $65(13 \times 5$ line $)$ nozzles.

Hirose Paper Co. Ltd. [20, 21] has proposed a nanofiber production technique characterized by electrospinning through applying the voltage that maintains nanofiber conditions in bubbles that are continuously generated in a polymer solution (Fig. 3). Consequently, it is necessary to efficiently repeat the generation and bursting of the bubbles formed on the surface of the polymer solution. As an example of this process implementation, the degree of saponification for the polyvinyl alcohol is a mole percent of 87.0-89.0 dissolved to water, and the prepared concentration of the polymeric solution is 20 mass \% (polymer solution).

Nonwoven fabric (nonwoven fabric produced by Hirose Paper Co. Ltd., 15TH145 ) is installed as a porous material for bubble generation in order to introduce this polymer solution into a cylindrical, stainless-steel vessel with a diameter $80 \mathrm{~mm}$, and a supply of compressed air is effected

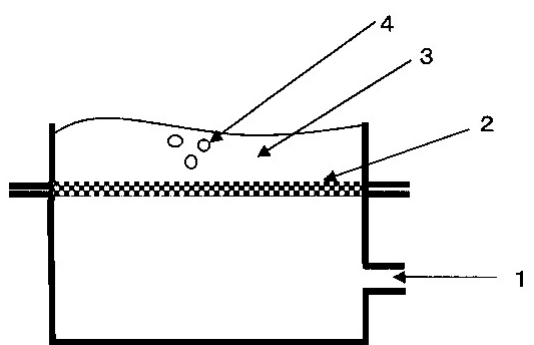

Fig. 3 Electrospinning device by bubble method (Hirose paper MFG Co. Ltd.) [21].

from the bottom. Compressed air at $4.0 \mathrm{kPa}$ is supplied through the nonwoven fabric, and foam is continuously generated along the entire surface of the polymer solution. Aluminum foil is positioned $8 \mathrm{~cm}$ from the surface of the bubbles as a counter electrode. Once the generation of bubbles on the polymer solution becomes uniform, a high voltage of $40 \mathrm{kV}$ is applied to the polymer solution, and the nanofiber is formed on the aluminum foil. It is desirable that the pressure of the compressed air is higher than pressure $P$ illustrated by the following equation.

$P=4 \times \gamma \times \cos \theta / D$

$\gamma$ is the surface tension of the polymer solution or the polymer melt.

$\theta$ is the contact angle of a porous material and a polymer solution.

$D$ is the largest pored diameter of the porous material.

Elmarco Co. Ltd. [22] has achieved continuous nanofiber production by placing multiple cylinder drums side by side.

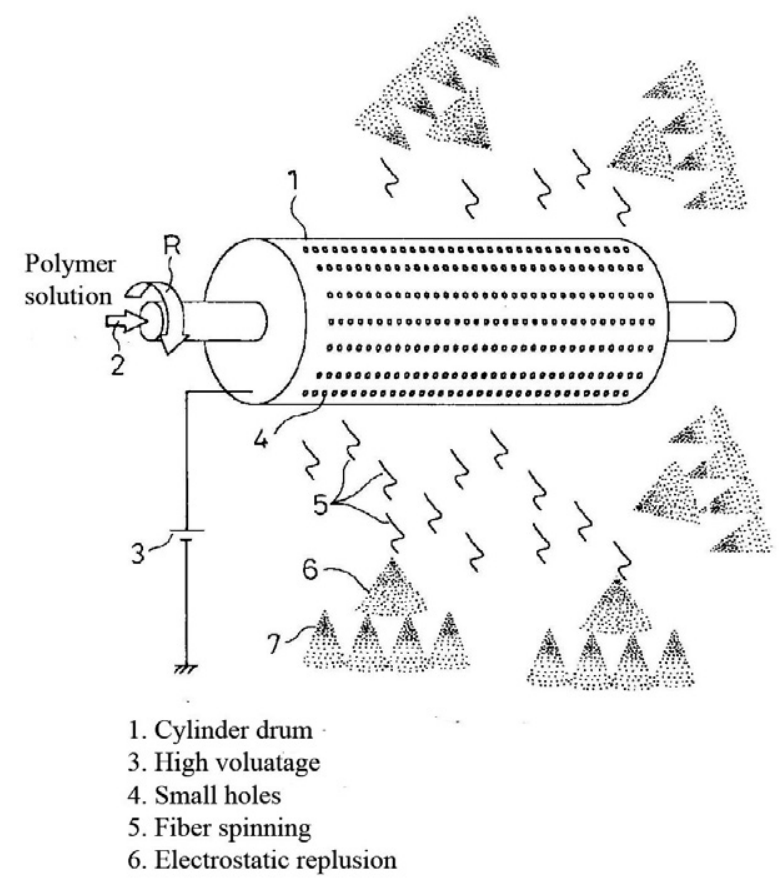

Fig. 4 Rotating drum type electro spinning device (Panasonic Co. Ltd.) [23]. 


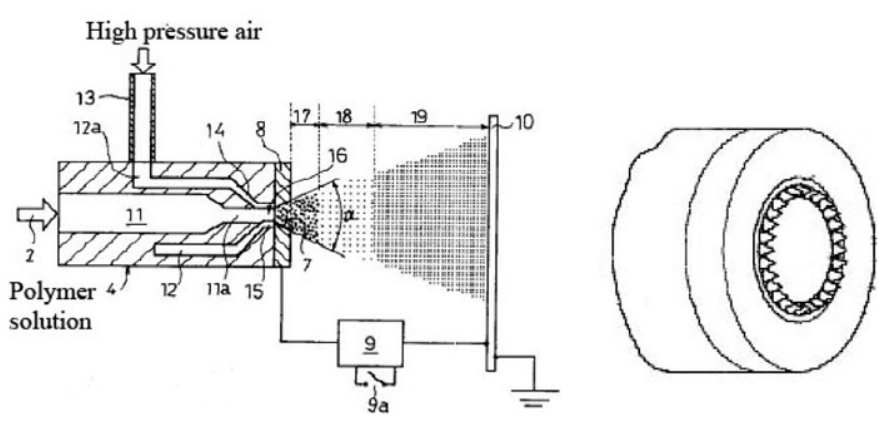

Fig. 5 Electrospray device that used high pressure (left) and its nozzle part (right) (Panasonic Co. Ltd.) [24]

For PVA and nylon, they are able to produce products similar to the nozzle method, but problems such as the countermeasures against sparks continue to be a problem in organic solvent systems.

Methods for improving productivity by increasing performance of a single spray nozzle by a factor of 50 of the current rate were tackled in the NEDO Project. This apparatus makes three-dimensional application possible. One device is a method of electrospinning from numerous holes on the drum surface. In the other device, high-pressure gas is used to atomize the polymer solution and electric charge concentrates on the protrusion of the nozzle. As a result, this device produces a nanospray by electrifying the atomized particles (Figs. 4, 5) [23, 24]

\section{2 Shape of nozzle}

The spinning part of the nozzle has not yet been clarified in electrospinning equipment for industrialization. Apparently another companies and research laboratories use the nozzle method, though Elmarco and Hirose Paper alone do not use nozzles. One drawback to the nozzle method is considerable time to wash the nozzle. The cleaning of the plugging of large number of nozzles is not easy. The cost of the nozzle might increase sharply though it is also possible to apply a protruding pin for washing the nozzle. Furthermore, another problem is the solidification of the polymer solution and adhesion of the nanofiber on the tip of the nozzle. It becomes, therefore, necessary to wipe the nozzle tip periodically. Wiping hundreds of nozzles, however, presents a herculean and expensive task.

And how about the flat-nozzles and the bubble method? The biggest task is preventing the evaporation of the solution in which the drum is immersed. Countermeasures to suppress steam pressure in the solvent area are required when organic solvent at a low boiling point is used. It is also said that the distribution of the fiber diameter of the nanofiber obtained by the drum method is wider than the nozzle method. In the flat system, the orientation of the
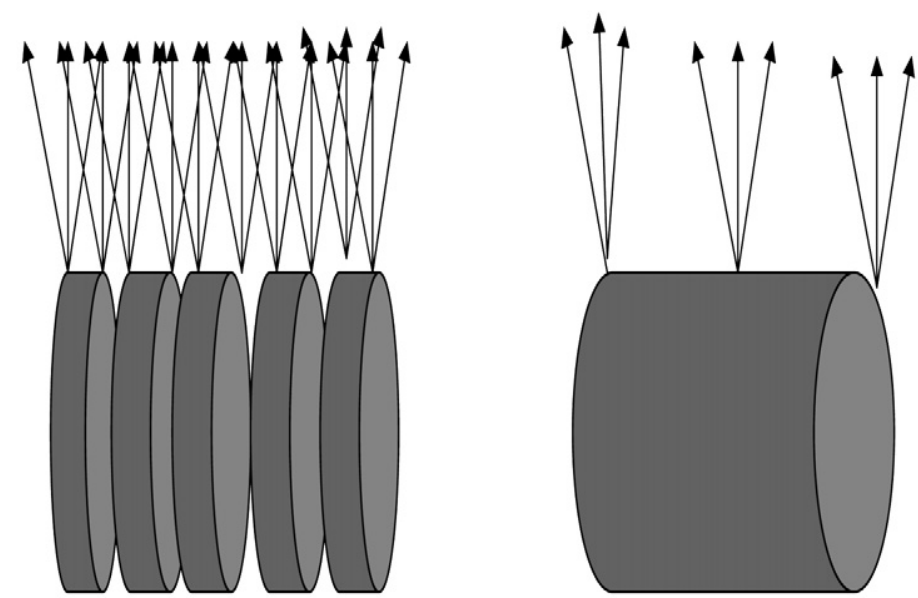

Fig. 6 Simulation of electrospinning from multi disk (left) and drum (right). Spray/spinning is generated from the edge of the drum, and the center part is not generated so much. For this reason, it is better to estimate using the muliti disk [25].

polymer molecule chain may be weaker than the nozzle system. Wang et al. [25] clarified that the electric charge concentrates on the edge portion of the drum and the electric charge at the center of the drum is weak by the electric field simulation. Wang asserted that a large number of thin disks would prove advantageous (Fig. 6).

\section{3 Solution delivery method}

Solution accumulates into the tank in the drum and the bubble method. It is a simple method since if it decreases all you have to do is add more solution. The solution is supplied with a syringe or through air pressure in the case of the nozzle system. Theoretically, air pressure is easier. However, since the concentration is $5-25 \mathrm{wt} \%$ (viscosity of 50-1000 mPa.s), the viscosity of the solution used in electrospinning is too low to be controlled by air pressure. There are also methods that use gear and tube pumps, but these pumps and tubes must be made with insulation materals. There is a danger of transmitting a charge through the solution and causing a spark in the pump because a high voltage is applied to solution. The advantage of the syringe system is that the solution supply is stable. However, a syringe pump is a batch type and needs to be stopped to supply more solution. Furthermore, when you increase the size of the syringe pump, it costs more because the pump mechanism also must be enlarged, and the cleaning in the syringe pump after use is also troublesome.

\section{4 Spark prevention measures}

Current does not flow because the insulation resistance of the air is large enough to stem the flow of a current with the 
voltage of $50 \mathrm{kV}$ between the nozzle and the target when no spinning is being carried out. The amperage increases with the quantity of nanofiber sprayed during electrospinning. It is, therefore, necessary to carefully monitor the amount of the current. Current increases rapidly with an increase in voltage in the amount of the current of $30 \mu \mathrm{A}$ or more, requiring the design of a manufacturing device that will never generate sparks.

\section{5 Ventilation and solvent recovery}

Recovery of evaporating solvent and monitoring of the concentration of it is necessary. Three elements (burning material, air, and an ignition source) are needed to cause explosions, and electrospinning intrinsically creates these three. It is, therefore, recommended that nitrogen gas be used to replace the atmosphere and a charcoal filter used to recover solvents.

\section{Approach to manufacturing apparatuses in Shiga Prefecture}

For the nozzle and drum method, the numbers of spinnings at one time remains limited. If a lot of nanofiber is spun at once, evaporation of the solvent will become insufficient and the adhesion of fibers and dissolution of nanofiber on the target will begin. Though the control of irregular thickness of nonwoven nanofiber is also important, it is still difficult. Thus it is necessary to disturb the nanofiber using such methods as those that vibrate the nozzle minute in order to equalize the non-uniform thickness of the nanofiber from the nozzle and methods for blowing air out from the nozzle. In Shiga Prefecture, the manufacturing equipment was developed through support from Ministry of Economy, Trade and Industry. These features are described in the following sections.

\section{1 Serial rolling-up equipment}

These provided facilities allow continued rolling of material at $60 \mathrm{~cm}$ in width and speeds $1 \mathrm{~cm} / \mathrm{min}-10 \mathrm{~m} / \mathrm{min}$ (Fig. 7(a), (b)).

\section{2 Resin nozzle}

Though metal nozzles are necessary for the manufacturing equipment, disposable resin nozzles are suitable for the cleaning of the nozzle by solution exchange and nozzle blockage in test machines for continuous production. We experimentally produced a multi-nozzle head with 10 nozzles positioned at $1 \mathrm{~cm}$-intervals. To make the quantity of delivery from each nozzle constant, a taper is applied to the internal resin pool part - the diameter in the nozzle is smaller at the center - and the exterior is enlarged. An inexpensive, commercial pipette chip can be attached to the nozzle tip, and this resin nozzle is scheduled for commercial sales. Furthermore, the resin nozzle, unlike its metal counterpart, can prevent nozzle dirt from attaching to the nanofiber suspended in air. We are planning to produce a $150 \mathrm{~cm}$-wide, $20 \mathrm{~cm}$-long base with 20 disposable nozzles (Figs. 8, 9).

\section{3 Syringe pump}

We have found that the use of a syringe pump as the technique for quantitatively supplying the polymer solution to each nozzle is most suitable. When the polymer solution is supplied to the multi-nozzle head through air pressure, there is a difficulty in setting the pressure to various solution

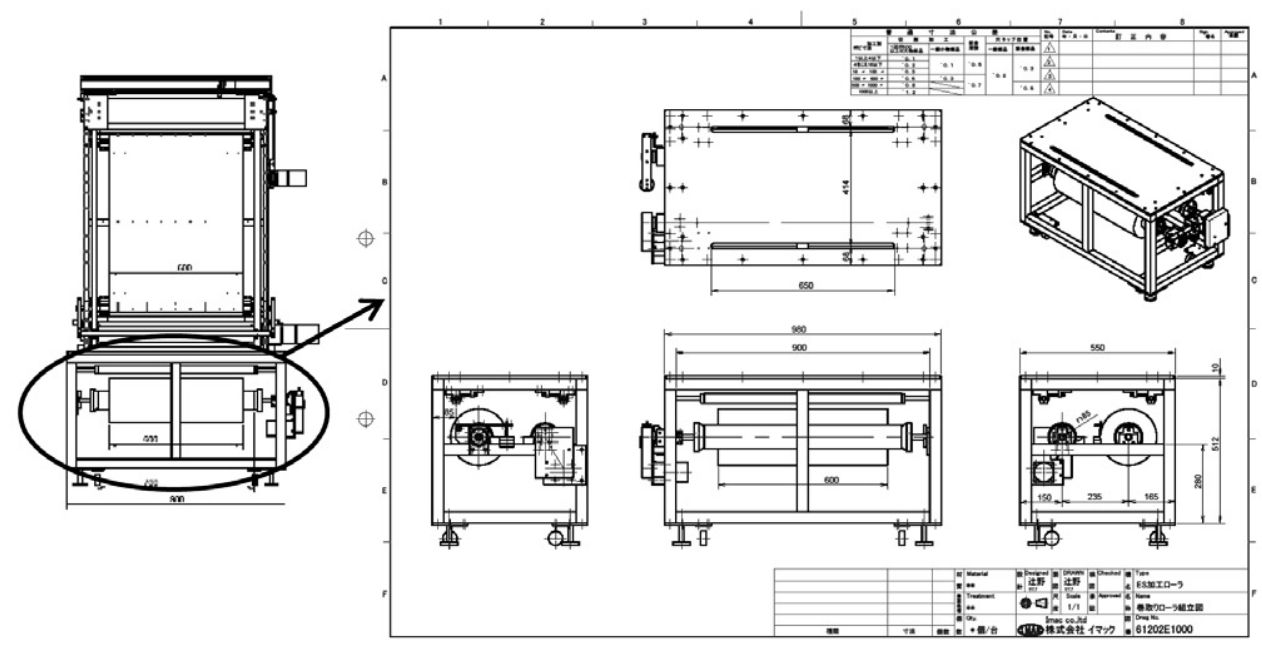

Fig. 7 (a) Schematic diagram of target part and sending off and take-up parts of cloth/film.

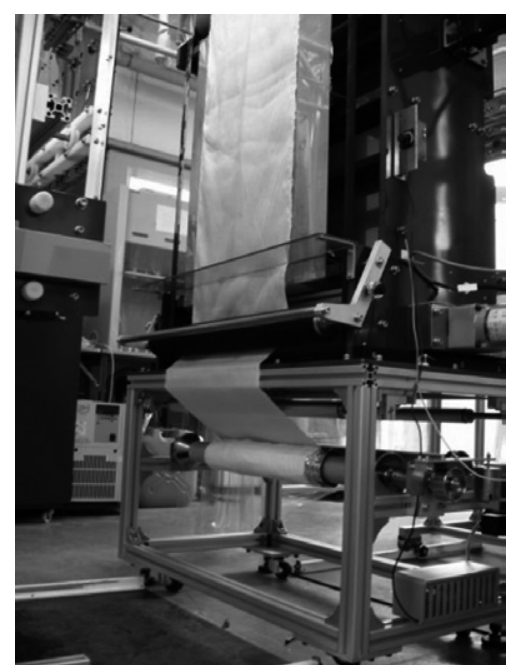

Fig. 7 (b) Photograph of continuous winder. 

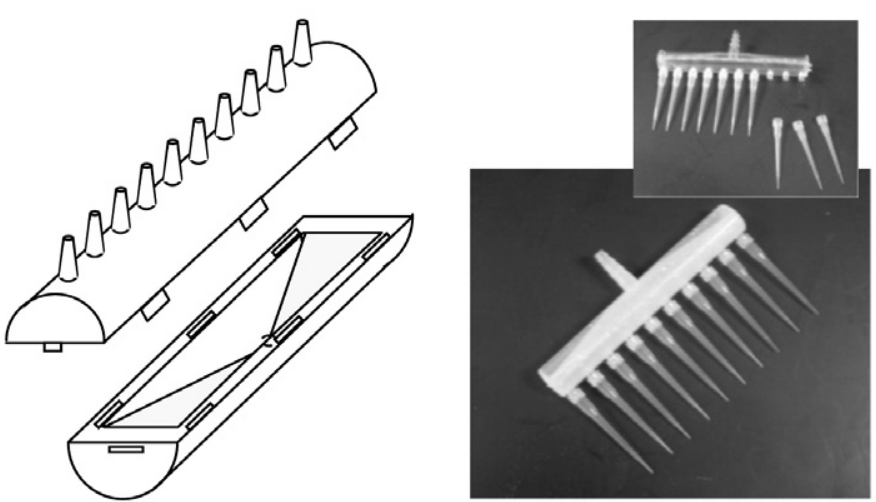

Fig. 8 Disposable multi resin nozzle (Installation of a disposable pipet is more possible in the nozzle tip).

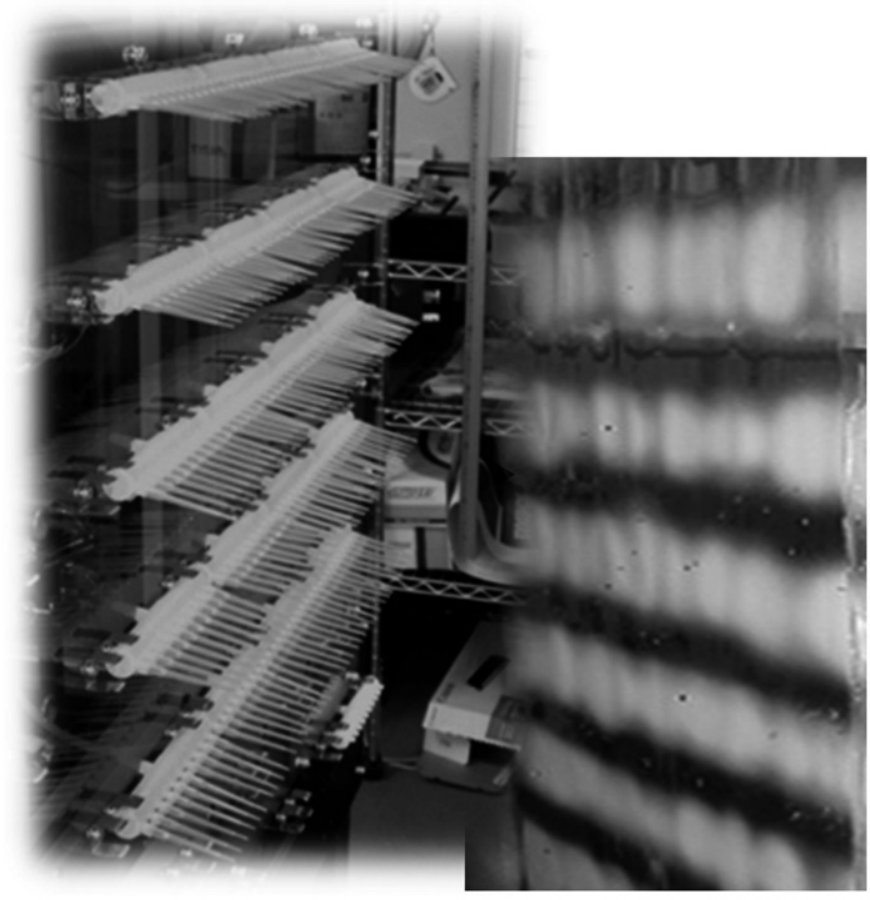

Fig. 9 Photograph of resin nozzle installed in vertical electro spinning machine.

viscosities. Since this device mounted in the vertical direction, we have supplied a syringe pump for the various controls.

\section{4 High speed camera}

The confirmation of nanofibers or nanoparticles cannot be judged without observation using a high magnification microscope. However, this is not easy to do in commercial manufacturing processes. It is also difficult to take samples from portions of production. For this reason, we have devised a method for watching the condition of the spinning from the nozzle employing a high-speed camera. The highspeed camera continuously observes the process, taking images at 1/1000s. Fig. 10(a) show images taken during the

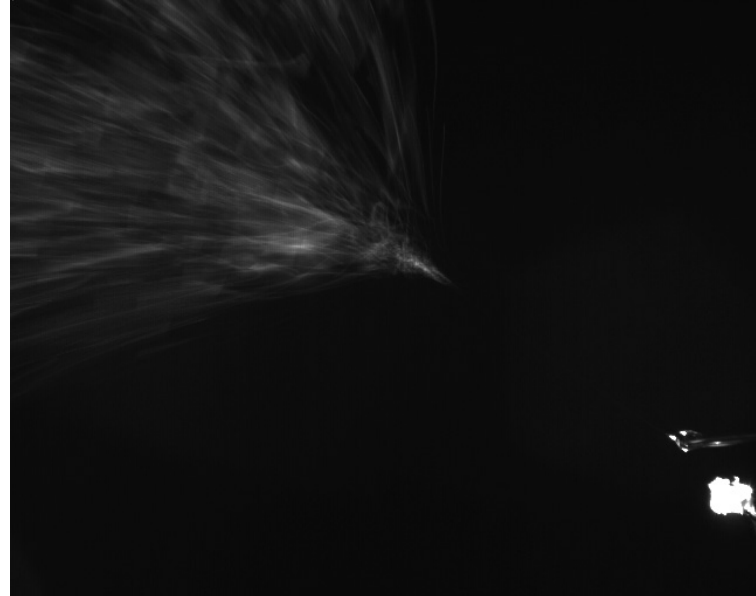

Fig. 10 (a) High speed camera image when nanofiber is made well (1/1000s).

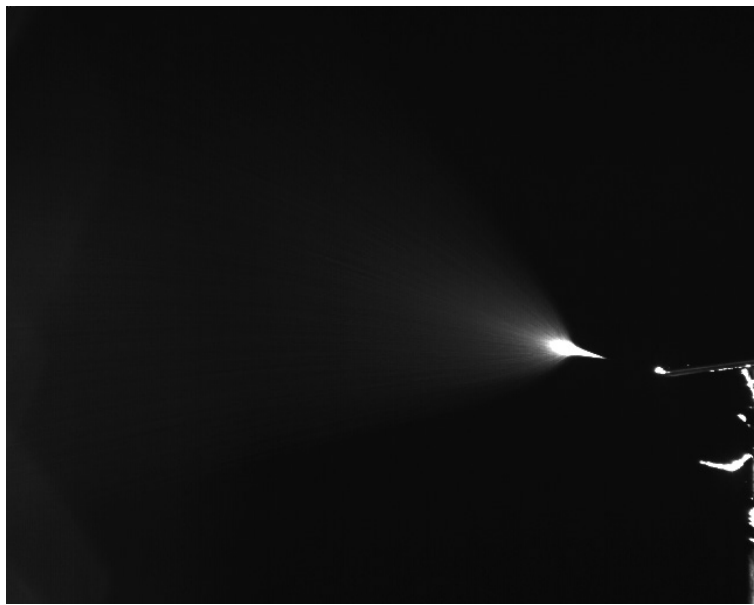

Fig. 10 (b) High speed camera image when nanoparticles cannot be made (1/1000s).

successful production of nanofiber. The length of the spray from the nozzle is long, and it is evident that a thin nanofiber is subsequently produced by electrostatic repulsion. On the other hand, Fig. 10(b) shows nanoparticle. The spray length from the nozzle tip to the electrostatic repulsion is short. The segmentation by the electrostatic repulsion is immediately generated, because the entanglement of the molecular chain does not occur with low concentration and low molecular weight. Moreover, the particle is being sprayed after the repulsion of static electricity.

\section{Concluding remarks}

The current state of the nanofiber research by the electrospinning method was reported. Nanofiber research and the processing technology in Japan have reached the stage which leads the world. Nanofiber manufacture by the electrospinning method will start soon by projects, such as 
NEDO and Shiga Prefecture, and application field of nanofiber that can be mass-produced are as follows; filter, medical, apparel and electronic industry material, etc.. In addition, polypropylene nanofiber of submicron became possible by melt electrospinning.

\section{References}

[1] Yamashita Y (2007) "Electorospinning - The latest in nanotechnology", Sen-i sha

[2] Ogata N, Shimada N, Yamaguchi S, Nakane K, Ogihara T (2007) J Appl Polym Sci, 105, 1127-1132

[3] Ogata N, Yamaguchi S, Shimada N, Lu G, Iwata T, Nakane K, Ogihara T (2007) J Appl Polym Sci, 104 ,1640-1645

[4] Ogata N, Iwata T, Yamaguchi S, Nakane K, Ogihara T, Lu G (2007) J Appl Polym Sci , 104, 1368-1375

[5] Takasaki M, Fu H, Nakata K, Ohokoshi Y, Hirai T (2008) Sen-i Gakkaishi, 64, 29-31

[6] Nakata K, Ohokoshi Y, Gotoh Y, Nagura M, Kim SH (2007) Sen-i Gakkaishi, 63, 307-312

[7] Uddin AJ, MashimaY, Ohkoshi Y, Gotoh Y, Nagura M, Sakamoto A, Kuroda R (2006) J Polym Sci Part B, 44, 398408
[8] Dalton PD, Grafahrend D, Klinkhammer K, Klee D, Möller M (2007) Polymer, 48, 6823-6833

[9] Ellison CJ, Phatak A, Giles DW, Macosko CW, Bates FS (2007) Polymer, 48, 3306-3316

[10] Kuo CC, Lin CH, Chen WC (2007) Macromolecules, 40, 6959-6966

[11] Korakot S, Neeracha S, Prasit P, Pitt S (2007) Polymer, 48, 1419-1427

[12] Santi T, Ittipol J, Pitt S (2007) Polymer, 48, 5030-5041

[13] Kyoshu University, JP2007-319074A

[14] http://www.donaldson.com/en/filtermedia/nanofibers/

[15] http://www.espintechnologies.com/company.htm

[16] http://www.finetextech.com/index_start.jsp

[17] http://www.awapaper.co.jp/index.html

[18] http://www.nano21c.com/

[19] http://www.nanostatics.com/index.htm

[20] http://www.hirose-paper-mfg.co.jp/el/el.html

[21] Hirose paper MFG, JP2008-25057A

[22] http://www.elmarco.com

[23] Panasonic, JP2008-31624A

[24] Panasonic, JP2008-43944A

[25] Niu H, Lin T, Wang X (2008) The fiber society 2008 spring conference, 15-16 